\title{
Optimization of Multijunction Solar Cells Through Indoor Energy Yield Measurements
}

\author{
Iván García, William E. McMahon, Myles A. Steiner, John F. Geisz, Aron Habte, and Daniel J. Friedman
}

\begin{abstract}
The variability of the solar spectra in the field may reduce the annual energy yield of multijunction solar cells. It would, therefore, be desirable to implement a cell design procedure based on the maximization of the annual energy yield. In this study, we present a measurement technique to generate maps of the real performance of the solar cell for a range of light spectrum contents using a solar simulator with a computer-controllable spectral content. These performance maps are demonstrated to be a powerful tool for analyzing the characteristics of any given set of annual spectra representative of a site and their influence on the energy yield of any solar cell. The effect of luminescence coupling on buffering against variations of the spectrum and improving the annual energy yield is demonstrated using this method.
\end{abstract}

Index Terms-Energy yield, luminescence coupling (LC), multijunction solar cell, variable spectrum.

\section{INTRODUCTION}

$\mathbf{M}$ ULTIJUNCTION solar cells take advantage of spectrum partitioning to minimize the thermalization losses and enable the highest efficiencies demonstrated so far. However, this is attained at the cost of a higher sensitivity to spectral variations. In the field, the solar spectrum changes over the course of the day and the year, with the air mass (AM) and the turbidity [or aerosol optical depth (AOD)] being the most influential parameters [1]. Both AM and turbidity cause an imbalance in the spectral content mainly by reducing the high-energy photon content (i.e., toward a top-cell current limited situation). As a consequence, the conversion efficiency of the cell is not optimal at all instants of the day and the year, reducing its total annual energy yield. The optimal approach to designing multijunction solar cells would be to optimize their performance for the variable spectra under which they are intended to operate in the field and not for a single reference spectrum.

Various approaches for energy yield prediction for multijunction solar cell-based CPV systems have been developed and tested by other authors. For example, in [2], the specific energy production rate measured outdoors for a particular location is used to predict the energy yield at other locations. Kinsey and Edmondson [3] theoretically predict the solar cell performance under different spectral irradiance conditions to obtain the energy yield for real atmospheric data and spectra sets. The measured quantum efficiency and the electrical parameters (open-circuit voltage) at one concentration were used to predict the performance of the multijunction cell for different sets of annual spectra. The methods presented in these works rely on outdoor measurements or on the calculation of the solar cell electrical performance for varying spectral conditions. Moreover, they do not take into account processes such as luminescence coupling (LC), which can introduce nonlinearities in the evolution of the short-circuit current with the spectrum and which affects the open-circuit voltage as well [4].

In this paper, we present a characterization technique that enables the indoor measurement of the performance of a multijunction solar cell for a wide range of sunlight spectral compositions and intensities. Solar cell performance maps are obtained and are used to determine the output of the solar cell for any given spectrum and to predict the annual energy yield of the solar cell device for a given set of annual variable spectra representative of a site.

In this experimental method, all of the internal processes in the solar cell when the solar spectrum changes are taken into account, including LC. LC in a multijunction solar cell takes place when an upper, higher bandgap subcell produces more photocurrent that the underlying subcells. The excess photogenerated carriers can recombine radiatively and be transferred to lower bandgap subcells, increasing their photocurrent. This, in turn, reduces the current mismatch and moves the bias point of the top cell towards a lower voltage, reducing its emission of photons. A steady state is reached [4], in which the bottom cell photocurrent is higher than its externally induced photocurrent, and therefore, the current in the series-connected multijunction solar cell is higher. It follows that LC is beneficial in currentmismatched situations, since it tends to correct the photocurrent imbalance in the subcells. LC serves as a buffering mechanism against performance losses in the multijunction cell due to the inevitable variation in the current matching across the solar cell junctions when the solar spectrum varies through the course of each day and year [5]. This should lead to an increased efficiency and overall yearly energy yield.

In the first part of this paper (see Sections II and III), twojunction (2J) solar cells with different degrees of LC are used to present the energy yield measurement method and illustrate its application to optimizing solar cells for maximum annual energy yield at 1 sun. In Section IV, a more complex 4J 
solar cell structure is used to analyze the effect of the spectral characteristics over a broader spectral range.

\section{Performance map Measurement Technique}

The measurement technique developed relies on the ability to generate a set of light spectra with a range of spectral compositions. A Xenon arc lamp is used in combination with adjustable high-power LEDs in order to achieve this. The LEDs are mounted in a collimator to minimize divergence and achieve a uniform spot of at least $2 \times 2 \mathrm{~cm}$. This is enough for our application, since the cells used are $0.3 \times 0.3 \mathrm{~cm}$. By individually adjusting the power of each LED, the current balancing in the multijunction cell is modified, mimicking spectral variations during operation of the cell in the field. A calibrated spectrometer is used to measure the Xe lamp + LED light spectra, and the spectral correction factor is obtained using reference isotype cells with equivalent response wavelength ranges so that the equivalent number of suns in each subcell can be determined for any spectral composition. The $I-V$ curve of the cell is measured for each case, and its performance parameters (shortcircuit current, open-circuit voltage, maximum power, etc.) are charted against the number of equivalent suns in each junction, similarly as in [6] and [7]. Note that this method is highly automatable. Moreover, if the spectral power of the Xe lamp and LEDs (or any other light source used) is sufficiently stable and accurately measured, no simultaneous measurement of calibrated reference cells is required.

Concerning the accuracy of this characterization method, several aspects are important. First, determining the light level (number of suns) for each subcell requires the external quantum efficiency (EQE) of the device under test and of the corresponding reference isotype cell as inputs. Absolute EQE measurements are usually difficult to obtain, but the result of the spectral correction factors and of the number of suns in each subcell is insensitive to any scaling of the $\mathrm{EQE}$ due to measurement errors [8]. Another possible source of error in the measurement is the uniformity and stability of the light sources. In our setup, we continuously monitor the Xe lamp power, and the LED sources have been found to be stable. Since the reference cells and the device under test are measured sequentially, attention must be paid to always place them at the same spot, to minimize the effect of possible nonuniformities in the light. To this end, we use a laser pointer that indicates the exact position to be used during measurements. Finally, the electrical measurement of the $I-V$ curves is carried out using the four-point probe technique, thus minimizing the error caused by the wires and contacts. In summary, the method is robust enough that its accuracy relies basically on the quality of the components used. In this paper, we have not carried out a detailed quantification of the measurement errors in our current setup, since the focus is on the method and illustrating its application.

As mentioned in Section I, in this paper, we analyze cells that exhibit LC. A brief explanation of the model we use to describe LC will be provided here; a more thorough explanation can be found in [4] and [9]. Two main parameters are used. First, the LC factor $\eta$ is the ratio of photocurrent generated by

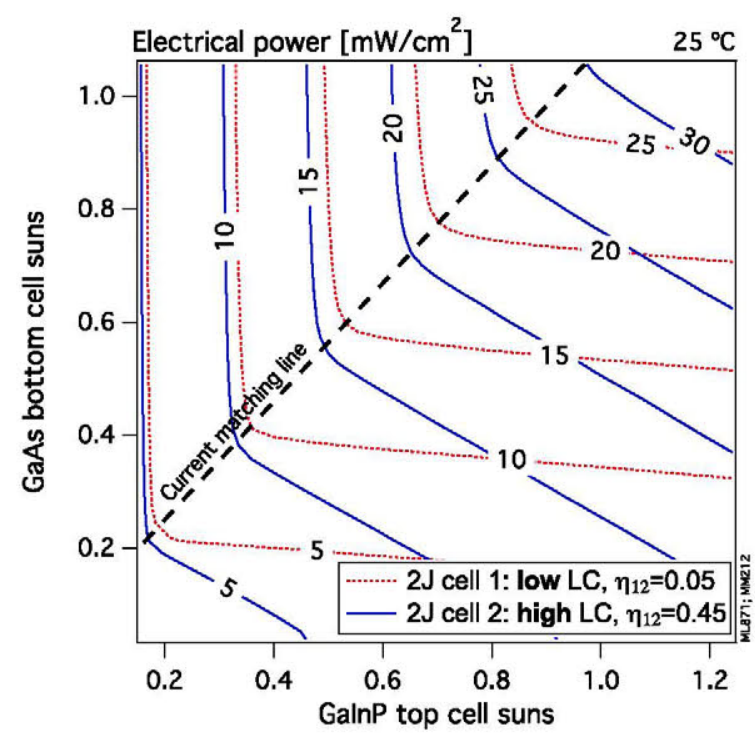

Fig. 1. Measured maximum power $\left(P_{\max }\right)$ maps for GaInP/GaAs 2J solar cells with two measured LC factors $\left(\eta_{12}\right)$ shown in the legend. The number of suns in each junction is referenced to the AM1.5-G173-direct spectrum at $1000 \mathrm{~W} / \mathrm{m}^{2}$. The map contains $40 \times 40$ measured points.

$\mathrm{LC}$ in the subcell absorbing the luminescence to the total $\mathrm{J}_{01}$ recombination in the subcell emitting the luminescence. Second, the $\varphi$ parameter in the subcell emitting the luminescence is defined as $\varphi_{i}=\mathrm{J}_{02} /\left(2 \sqrt{ } \mathrm{J}_{01}\right)$ and represents the linearity of the $\mathrm{LC}$ in our model. Since only the $\mathrm{J}_{01}$ recombination gives rise to luminescence, the intensity of the $\mathrm{LC}$ is higher when this $\mathrm{J}_{01}$ recombination component dominates over the $\mathrm{J}_{02}$ component. Both the LC factor $(\eta)$ and the $\varphi$ parameter describe the intensity of the LC effect and its variation with the injected current. The $\eta$ and $\varphi$ in a mutijunction solar cell can be measured following the method described in [4], and this method was used to measure the LC parameters of the experimental cells used in this study.

Fig. 1 shows representative performance maps obtained for similar inverted GaInP/GaAs 2J solar cells with different magnitudes of LC as quantified by the LC factor $\left(\eta_{12}\right)$ [4]. The different LC factors are obtained by using GaInP top cells with different internal luminescence efficiencies as their junction position is changed, as described in [10]. Iso- $P_{\max }$ contours are plotted against the equivalent number of suns in each subcell. The dashed line corresponds to the condition when the $2 \mathrm{~J}$ cell is current matched and lies at the points where the iso-maximum power contours bend. A more accurate positioning of this line can be determined by plotting a map of the fill factor (FF), which is not shown for brevity: The current-matching line corresponds to the minimum in the FF contours. If the GaAs bottom cell light intensity (suns) is increased above the current-matching line, while holding the GaInP top cell intensity constant, the $P_{\max }$ increases slightly at the beginning due to a higher FF and then remains constant in both tandem cell cases. The effect of the $\mathrm{LC}$ is clearly observed in the opposite case corresponding to the region to the right of the current-matching line, i.e., where the top cell is overdriven with respect to the bottom cell. For the cell with high $\eta_{12}$, the iso- $P_{\max }$ contours are inclined so that increasing 
the number of suns in the top cell, while keeping constant the number of suns in the bottom cell, gives an increased $P_{\max }$. This type of measured-performance contour plot can be used as quantitative "fingerprints" of the real performance of the multijunction solar cell for a range of spectral compositions. These data can be collected for cells with any number of junctions; for the case of a 2J solar cell, these fingerprints can also be easily represented visually as a contour plot with the suns of the two junctions as independent variables, as shown in Fig. 1. For higher number of junctions, a performance contour plot including all subcells at the same time is not possible, but the contours of individual pairs of junctions can be plotted the same way in order to obtain a similar visual aid, as will be shown in Section IV.

The energy yield of the solar cell for a particular site, for the set of solar spectra considered, can be calculated as the summation of the energies generated for each spectrum in the spectra set. These energies are computed by extracting the power for each spectrum from the cell's $P_{\max }$ map, multiplied by a corresponding time to produce an energy, then summing the energies to compute the total energy for the entire set of spectra. This method provides a convenient and repeatable method of assessing the energy yield for any annual solar spectra set available.

The experimental results presented in this paper, which illustrate the method developed to measure the energy yield indoors, are obtained for a constant temperature of $25^{\circ} \mathrm{C}$. In the field, however, the operating temperature of solar cell receivers is not constant and varies throughout each day and year as atmospheric conditions change. These changes in temperature significantly affect the calculation of the energy yield, mainly due to the effect of the temperature on the $V_{o c}[11]$. Other authors have taken this into account by modeling the variations in the solar cell parameters with the temperature [3], [12]. In our approach, changes in performance with temperature can be directly measured by including temperature variations as an additional dimension in the measured solar cell performance datasets. Note that, technically speaking, obtaining the required additional data for different temperatures can be easily automated: A temperature-controlled sample holder can be used during the measurement of the $I-V$ curves at different spectral contents.

Another important issue is the application of this method to concentrator operation. In this paper, the experimental results obtained are for 1-sun operation. Obtaining this type of performance maps is time consuming and requires being able to generate a wide range of spectral compositions in the light. The possibility of compressing the whole annual spectra set of a site into a small representative set of "proxy" spectra is being studied now. A judicious reduction in the number of required spectra can reduce the total measurement time and energy yield calculation to a matter of seconds. In addition, it would facilitate indoor energy yield measurements under concentration, which are affected by the fact that controlling the light spectrum in a flash-lamp setup is complicated. Therefore, it would be difficult to generate all of the spectra used in the performance maps, but generating a few spectra in a flash simulator is feasible. We have obtained promising results, in terms of energy yield calculation accuracy, by applying an AM binning algorithm

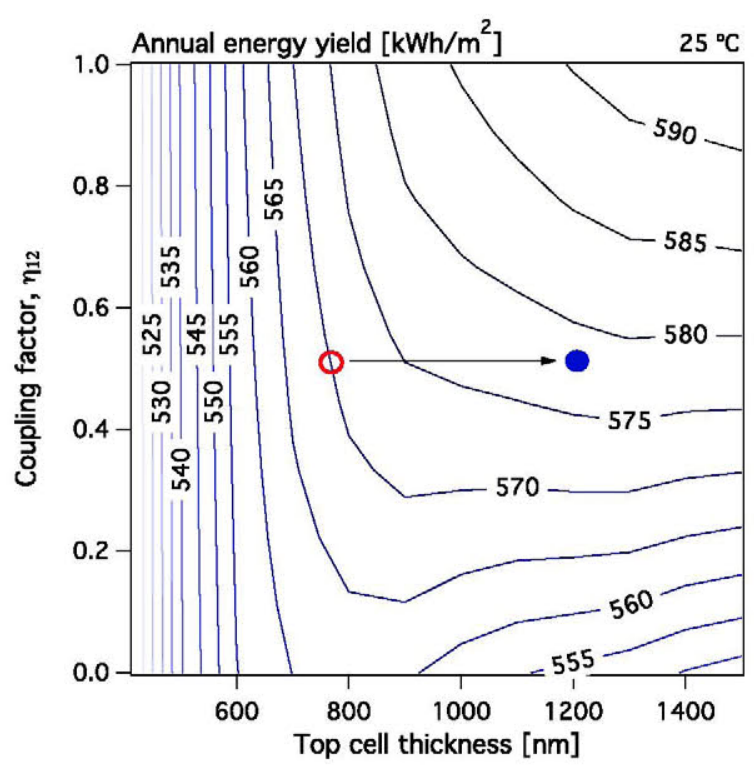

Fig. 2. Annual energy yield of a GaInP/GaAs tandem solar cell for a range of top cell thicknesses and LC factors $\left(\eta_{12}\right)$. The solar spectra used were obtained using the TMYspec model and measured broadband parameters for Golden, CO, USA, in 2012. The empty (red) and filled (blue) dots indicate the cases further analyzed in the top and bottom parts of Fig. 3, respectively.

to the Golden 2012 spectra set for GaInP/GaAs-based multijunction solar cells. As few as six spectra have been found to be sufficient to represent the whole year, with a deviation in the calculated energy yield of less than $0.5 \%$ with respect to the results obtained considering the whole set of annual spectra. However, the robustness of the method when applied to various spectra sets for different solar cell configurations and levels of LC, must still be tested; this will be the topic of a follow-up paper.

\section{ENERGY Yield AND Optimum Cell. Design}

An illustrative example of the optimal design of a multijunction solar cell for maximizing the annual energy yield is presented here, using a 2J solar cell. A set of inverted GaInP/GaAs tandem cells with varying top cell thickness but roughly the same $\eta_{12}$ (around 0.45) and recombination currents were used. The details of this set of cells were presented in [13]. Hourly-binned spectra for the Golden, CO, USA, site in 2012 were generated using the TMYspec model and measured broadband parameters [14]. More details about this kind of spectra and their influence on the energy yield calculations are presented in Section IV. For this cell design demonstration, the spectra used are a representative example for illustrative purposes and should not be used to draw general quantitative conclusions about energy production in Golden.

In Fig. 2, the calculated annual energy contours are plotted against the top cell thickness and $\eta_{12}$. These energy contours were obtained using power maps generated using the analysis described in [9]. All parameters used in the model correspond to the experimental cells described above, except that values of $\eta_{12}$ ranging from 0 to 1 were considered. The effect of the LC becomes apparent in this graph: As $\eta_{12}$ increases, the optimum top cell thickness to optimize the energy yield increases, as 

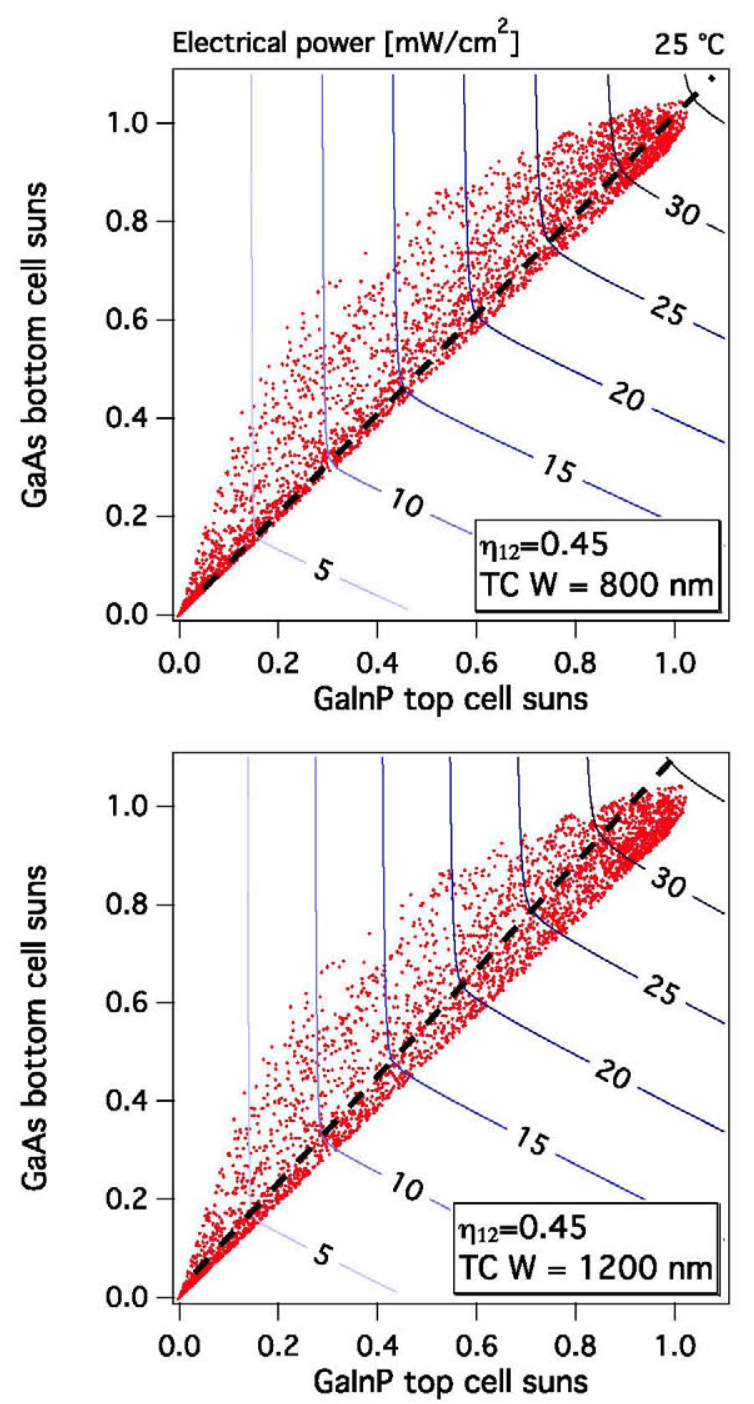

Fig. 3. Calculated $P_{\max }$ contours for tandem solar cells with two top cell thicknesses indicated in Fig. 2. Each red dot corresponds to the spectrum representing a particular hour during the year 2012 in Golden, CO, USA. The number of suns in each junction is referenced to the AM1.5-G173-direct spectrum at $1000 \mathrm{~W} / \mathrm{m}^{2}$.

predicted in [9]. For these particular spectra, if the top cell thickness is modified from its optimum for the AM1.5D-G173direct spectrum (around $800 \mathrm{~nm}$ ) to the optimum for maximum energy yield, improvements of $\sim 1 \%$ and $\sim 2 \%$ are obtained for tandem cells with $\eta_{12}$ of 0.5 and 1.0, respectively. Moreover, the total energy yield increase between optimized cells with $\eta_{12}$ of 0 and 1 is as high as $\sim 5 \%$, as shown in Fig. 2.

In Fig. 3, iso- $P_{\max }$ contours for the two experimental tandem cell cases indicated in Fig. 2 are plotted. The dashed line indicates current-matching between subcells and each red dot corresponds to a spectrum in the yearlong dataset- the characteristics of the resulting scatter plots will be further analyzed in Section IV. The highest power-producing spectra correspond to the region in the scatter plot around 1 sun in both junctions. In the thick top junction case, this region lies below the currentmatching line, where LC helps mitigate the effect of spectral variations. Conversely, when the top cell is designed for cur- rent matching under the reference spectrum, it operates most of the time in a top junction-limited regime, where the LC does not help reduce the effect of the spectrum variations. This explains the results shown in Fig. 2 and illustrates how plotting these $P_{\max }$ contour and the solar spectra is a powerful way of understanding the influence of the combined cell and spectral characteristics on the energy yield. Similar plots for any kind of cell and spectra can be used as a guide for designing solar cells that maximize the annual energy yield for a particular site.

\section{Application to Cells With More Than Two JUNCTIONS AND A BROADER SPECTRAL RESPONSE}

The GaInP/GaAs 2J solar cell case presented in the previous section illustrated the mechanics of the characterization technique and its application to energy yield prediction and design of the solar cell. However, state-of-the-art III-V multijunction solar cells comprise a higher number of junctions that extend the useful spectral wavelength range to longer wavelengths. Furthermore, the spectral variations due to changing atmospheric conditions are not uniform across the wavelength range. Therefore, the effect of these spectral variations on the performance of cells responding to a wider wavelength range may be significantly different from for case of a $\mathrm{GaInP} / \mathrm{GaAs} 2 \mathrm{~J}$ solar cell. In this section, we will use an experimental state-ofthe-art GaInP/GaAs/GaInAs $(1 \mathrm{eV}) / \mathrm{GaInAs}(0.7 \mathrm{eV}) 4 \mathrm{~J}$ invertedmetamorphic (IMM) solar cell to measure $P_{\max }$ maps, calculate the annual energy yield, and show the effect of the spectral variations for the wavelength ranges corresponding to each junction.

\section{A. Characteristics of Four-Junction Cell $P_{\max }$ Maps}

The 4J cells used are IMM devices with two metamorphic 1 - and 0.7-eV GaInAs subcells [15]. The useful wavelength range for this cell is $\sim 300$ to $\sim 1850 \mathrm{~nm}$. These devices exhibit strong luminescence, even in the metamorphic junctions, as shown in [15]. The $P_{\max }$ maps for these devices were taken by automatically sweeping the concentration in each junction from 0 to 1.2 suns (referenced to the AM1.5 G173-direct spectrum at $1000 \mathrm{~W} / \mathrm{m}^{2}$ ) in six steps, which gives a total of $6^{4}=1296$ $I-V$ curve measurements. Our energy yield calculations will once again utilize the hourly binned spectra for the Golden site in 2012 generated using the TMYspec model and measured broadband parameters [14]. The $P_{\max }$ maps obtained are shown in Fig. 4, plotted as blue contours against the number of suns in each pair of subcells (the other subcells not represented in each graph are held at approximately 1 sun). In these graphs, each colored dot represents the spectrum corresponding to a particular hour in the year-long dataset.

Compared with the 2J solar cell case, the shape of the contours for the $4 \mathrm{~J}$ device contains more complex and intricate information about the LC and the current-limiting junctions in each case. The slope of the current-matching lines (dashed lines in the plots) and their crossing points with the axis are affected by the current matching and LC. A current-matching line that does not cross the origin reveals that one subcell is absorbing luminescence from other subcell in the structure, i.e., that LC is 


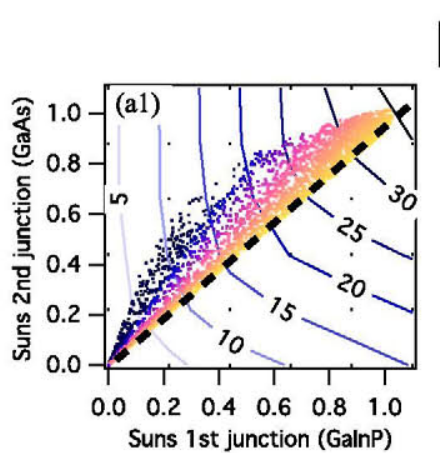

1 st junction vs. 2nd, 3rd and 4th junctions
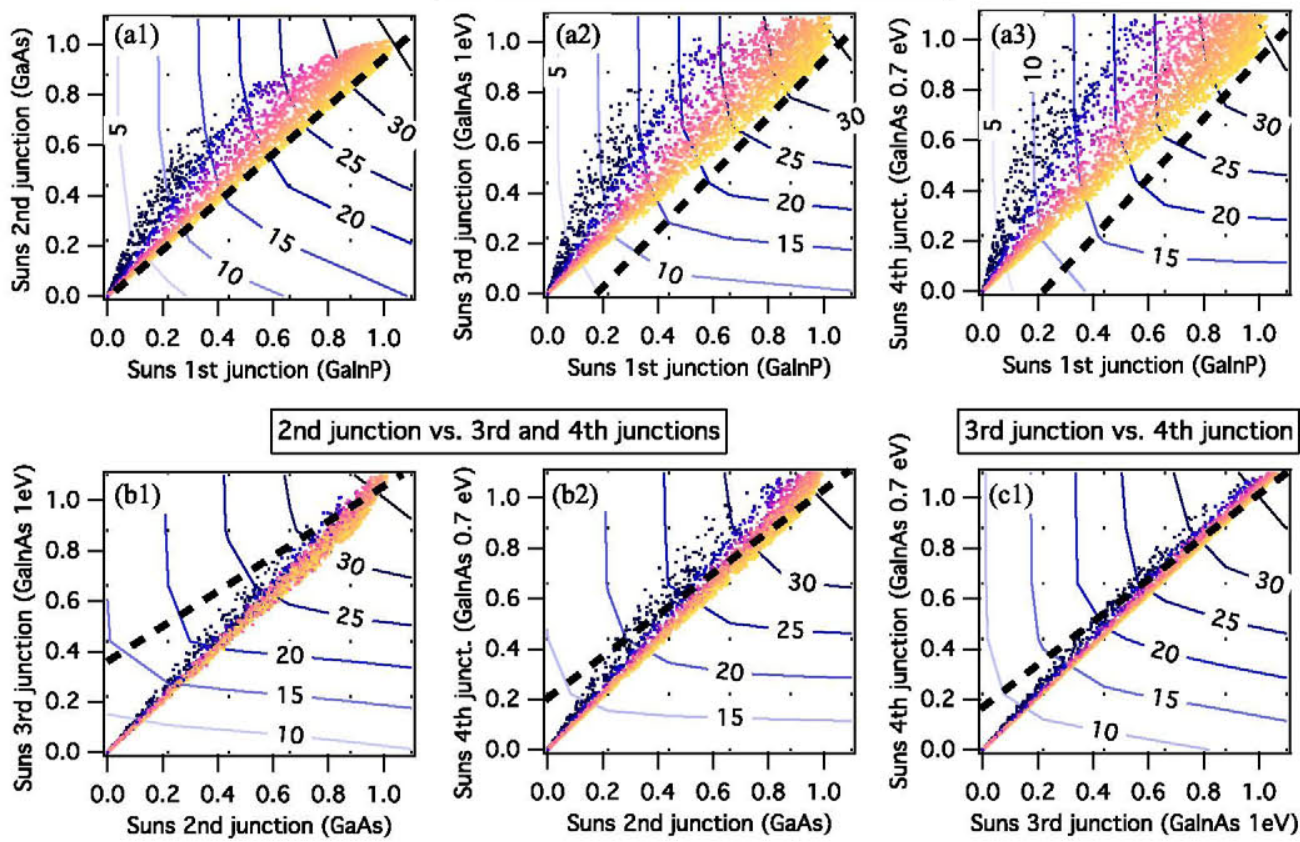

Contours: electrical power $\left[\mathrm{mW} / \mathrm{cm}^{2}\right]$

- - - current matching lines

Scatter plot:

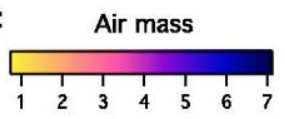

\begin{tabular}{c|c|c|c|}
\multicolumn{4}{c}{ 4J cell measured characteristics } \\
Junction & $\begin{array}{c}\text { 1-sun Jsc } \\
{\left[\mathrm{mA} / \mathrm{cm}^{2}\right]}\end{array}$ & $\eta_{\mathrm{L}, \mathrm{l}+1}$ & {$\left[\mathrm{~mA}^{1 / 2} \mathrm{~cm}^{-1}\right]$} \\
\hline 1st - GalnP & 12.2 & 0.53 & 0.32 \\
2nd - GaAs & 12.5 & 0.25 & 1.90 \\
3rd - GalnAs (1eV) & 13.3 & 0.22 & 0.63 \\
4th - GalnAs $(.7 \mathrm{eV})$ & 12.0 & $\mathrm{n} / \mathrm{a}$ & 1.90
\end{tabular}

Fig. 4. Measured $P_{\max }$ contours for a GaInP/GaAs/GaInAs $(1 \mathrm{eV}) / \mathrm{GaInAs}(0.7 \mathrm{eV}) 4 \mathrm{~J}$ solar cell. Each plot represents the variation of the $P_{\max }$ when the concentration in two subcells is changed with the other two subcells held at 1 sun. The number of suns in each junction is referenced to the AM1.5-G173-direct spectrum at $1000 \mathrm{~W} / \mathrm{m}^{2}$. The dashed lines correspond to the concentration pairs that produce current matching between the subcells. Each colored dot corresponds to the spectrum representing a particular hour during the year 2012 in Golden, CO, USA. The color of each dot represents its AM (see color scale below the graphs). The table shows the measured characteristics of the 4J solar cell, including the 1-sun short-circuit current ( $\left.\mathrm{J}_{\mathrm{sc}}\right)$ and the LC parameters $\eta$ and $\varphi$ described in Section II.

taking place. For example, in graph (a1), the current-matching line crosses the origin because when both first and second junctions are at 0 suns, there is no external or $\mathrm{LC}$-induced photocurrent in any of them. At the origin of graph (b1), there is no external source of photocurrent for the second and third junctions, but the cascading of LC from the first to second to third junctions generates a photocurrent in the second and third junctions, shifting the current-matching line. Similar reasoning can be used to understand the remaining graphs.

A slope of the $P_{\max }$ contours away from the current-matching lines indicates the existence of $\mathrm{LC}$ between the two subcells represented in the plot. The magnitude of this slope is linked to the LC parameter $(\eta)$ and the $\varphi$ factor, explained in Section II [4]. The table included in Fig. 4 shows the measured externally induced short-circuit current $\left(\mathrm{J}_{\mathrm{sc}}\right), \eta$ and $\varphi$ of the $4 \mathrm{~J}$ solar cell under analysis. A clear relationship between the parameter values shown in the table and the slope of the contours in graphs (a1) to (c1) can be observed. For example, the highest $\eta$ in the table, corresponding to the LC between the first and second junctions, gives rise to a higher slope in the contours in graph (a1). Note that graphs (a1), (b1), and (c1) show the coupling between adjacent junctions in the $4 \mathrm{~J}$ structure. The slope in the other graphs corresponds to the cascading coupling occurring when a subcell absorbs luminescence from overlying subcells and then re-emits photons that are subsequently absorbed by underlying subcells.

\section{B. Characteristics of Spectra Datasets}

The colored dots in graphs (a1) to (c1) of Fig. 4 represent occurrences of the corresponding pairs of subcell concentrations for the same Golden 2012 TMYspec-generated spectra used before. It is important to emphasize once again that we are using this set of spectra to approximate the spectral variations during a typical year in Golden and not to make quantitative predictions of performance for this particular site. Two impor- 

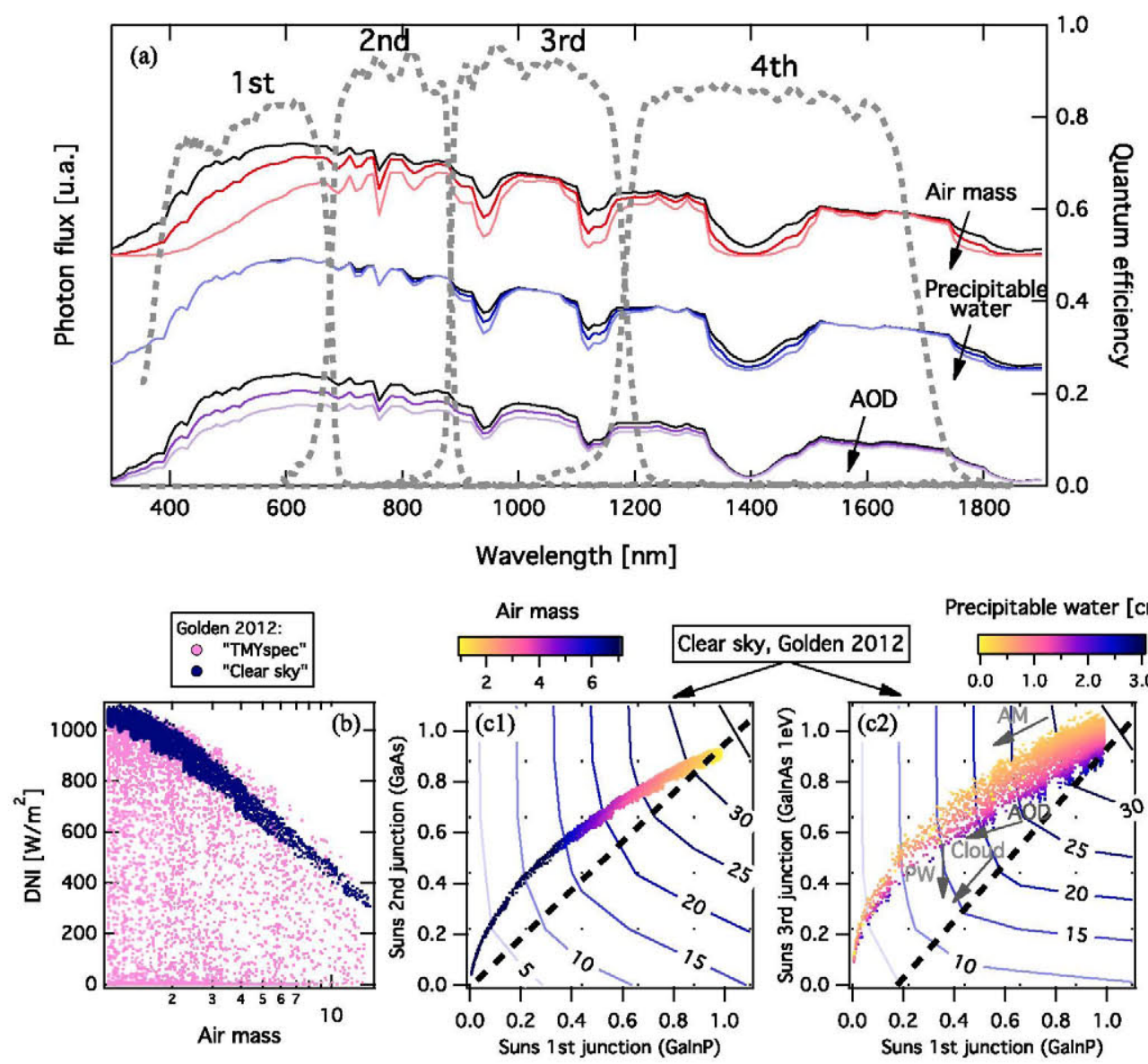

Precipitable water $[\mathrm{cm}]$
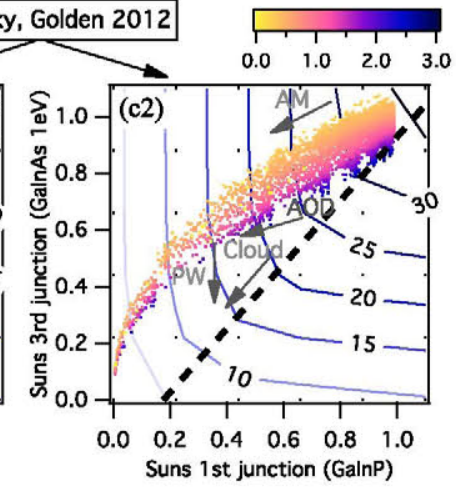

Fig. 5. (a) SPECTRAL 2-calculated direct spectra for varying AM, precipitable water, and AOD. The spectra are plotted using arbitrary units and are shifted vertically for readability; in the same graph, the $\mathrm{EQE}$ of the $4 \mathrm{~J}$ solar cell used is plotted. (b) DNI versus $\mathrm{AM}$ occurrences during the year for a set of spectra calculated using broadband parameters for Golden, CO, USA, in 2012; light dots are for spectra calculated using the TMYspec model including clouds and dark dots are for spectra calculated using SPECTRAL2 without clouds. (c1) and (c2) Occurrences of the "clear-sky" spectra set plotted on two P max contour plots corresponding to the 4J solar cell (see Fig. 4). The color of the dots represents the AM [graph (c1)] or the PW [graph (c2)]. The arrows in graph (c2) indicate how a dot would move if the parameter indicated increased.

tant characteristics are of relevance for the energy yield application discussed here. First, a significant dispersion in the points in the scatter plot is observed. Second, the shape and dispersion of the scatter plot changes significantly from graphs (a1) to (c1). Also note how a wide range of different subcell concentrations are produced for the same AM (indicated by the color of the dots). These characteristics can be understood with the help of Fig. 5 .

The top graph in Fig. 5 shows the direct spectrum and its qualitative variation as the $\mathrm{AM}$, precipitable water vapor $(\mathrm{PW})$, or AOD changes. In the same graph, the EQE of the 4J solar cell is plotted to show which junctions are mostly affected by the changes in the spectrum. The AM affects much more strongly the top cell than the other subcells. The PW affects only the third and fourth junctions. The AOD affects more the first and second subcells than the other subcells.

Another parameter affecting the arrangement of the scatter plots in Fig. 4 is the cloud cover. To analyze the effect of the clouds, we computed a set of spectra for the Golden latitude, for the same broadband atmospheric parameters as in the spectra of Fig. 4, using SPECTRAL2 [16] but assuming a clear sky for all spectra. We will call these two spectra "TMYspec" and "clearsky" hereinafter. The results are displayed in Fig. 5(b), (c1), and (c2). Fig. 5(b) shows a scatter plot of the direct normal irradiance (DNI) against the AM for both the "TMYspec" (light dots) and the "clear-sky" (dark dots) spectra. The DNI goes down as the AM increases, as expected. The atmospheric parameters, namely PW and AOD, cause a variation in the DNI for the same AM, which gives rise to the band-shaped scatter plot for the "clear-sky" spectra in graph (b). With AOD and PW set to 0 , the shape would be a line, since only the AM would affect the DNI. For the "TMYspec" spectra, however, the data in graph (b) are much more dispersed due to the treatment of the clouds by the spectral dataset and TMYspec model. To a first-order approximation, clouds block the direct sunrays and the DNI should go to 0 when they are present. Then, for the TMYspec spectra, which includes clouds, we would expect to see a band-shaped arrangement in the scatter plot of graph (b), corresponding to the no-cloud occurrences, and a cluster of dots around the horizontal axis, corresponding to 0 DNI when clouds are present. However, the TMYspec spectra used are binned in timespans of $1 \mathrm{~h}$. In the most frequent situations where the direct 
beam is blocked by clouds only during a fraction of the hour, the model scales the spectrum so that the resulting total DNI for the whole hour is the average. This produces the correct total irradiance for each hour, but a partly cloudy hour will be represented by a single spectrum with an average (for the hour) DNI power. This explains the (unphysical) scatter for the TMYspec spectra in Fig. 5(b) and is responsible for most of the scatter in the data of Fig. 4 as well.

Fig. 5(c1) and (c2) are equivalent to Fig. 4(a1) and (a2) but with scatter plots generated using the "clear-sky" calculated spectra. The main difference is that the scatter is vastly reduced for clear sky spectra, for the reason explained above. In Fig. 5(c1), the color of the dots represent the AM. In this case, the AM has a large impact in the shape of the scatter plot, because it affects the first junction more strongly than the second junction, as is apparent in Fig. 5(a). This is the reason for the curved shape of the scatter plot: As the AM increases, the number of suns in the first junction decreases faster than the number of suns in the second junction, producing the concave shape. Fig. 5(c2) follows the same scheme but this time looking at the first and third junctions. The color of the dots represents the PW in this case. As shown in Fig. 5(a), PW affects mostly the third and fourth junctions. Therefore, in this graph, the effect of both AM (which affects mostly the first junction) and PW (which affects the third junction) can be seen: The AM causes the curved shape of the scatter plot, while the PW gives rise to a dispersion of the dots toward a lower number of suns in the third junction. Similar reasoning can be applied to understand how the atmospheric parameters affect the number of suns in all pairs of junctions in the 4J solar cell, as shown in Fig. 4. The arrows in Fig. 5(c2) indicate (approximately) how a data point dot at that position would be affected by an increased value of the atmospheric parameter associated with the arrow.

For partly cloudy hours, the average DNI will be less than for a cloudless hour. Reducing the DNI while leaving the spectral content unchanged shifts the points toward the origin, filling in the concavity below the arcs in the various scatter plots (compare Fig. 5(c1) with 4(a1) or Fig. 5(c2) with 4(a2), for example). The impact of these partly cloudy spectra on energy-yield calculations is currently being investigated.

\section{Energy Yield and Influence of Luminescence Coupling}

Once the characteristics of a spectral dataset are understood, we can revisit Fig. 4 to analyze their effect on energy yield and to what extent $\mathrm{LC}$ can counteract them. The extent to which $\mathrm{LC}$ can mitigate losses due to spectral variations depends on the shape and position of the spectral data scatter plots relative to the $P_{\max }$ maps of the solar cell, as shown in Section III for the case of a $2 \mathrm{~J}$ solar cell.

Any AM or atmospheric change implies a variation in the spectrum, but its effect on the solar cell energy yield can be minimized in certain cases if $\mathrm{LC}$ is taken advantage of, as shown in Section III. Buffering by LC can only occur when a higher bandgap junction is overdriven with respect to a lower bandgap junction during the atmospheric changes, as shown in Fig. 3 for the 2J case. In an ideal situation with LC factors of 1 , the current-matching lines in optimum $4 \mathrm{~J}$ cell designs would be to the left of the spectra data scatter plots. In practice, coupling factors are below 1, meaning that not all excess photocurrent in the upper junctions is transferred as luminescence to the lower junctions. Therefore, LC can typically compensate for only a fraction of any current imbalance. This means that the design of the $4 \mathrm{~J}$ subcells must be such that the current-matching lines in the graphs of Fig. 4 cross the scatter plots. The exact position of these current-matching lines for optimal energy yield can be determined by using the same kind of approach as in Fig. 2. It is important to point out that the III-V multijunction solar cells used in this example are designed so that all junctions but the first (GaInP) are optically thick. Therefore, in this case, the approach of thickening the junctions to optimize the buffering provided by the LC is only useful for the first-second pair of junctions. The remaining alternative is redesigning the bandgap combination in the 4J structure, using the guidance of the performance maps.

\section{CONCLuSion}

A measurement technique has been implemented which uses performance maps to characterize and assess operation of multijunction solar cells under various solar spectra. The applicability of this method is illustrated by designing a 2J solar cell for maximum energy yield, taking into account the effect of LC. Although the specific results are only applicable to the cells and spectra used here, significant annual energy yield gains are obtained, providing an indication about the expected benefits of $\mathrm{LC}$ for other cases. The performance maps are also a powerful tool for assessing the impact of the various characteristics of the annual solar spectra used on the solar cell energy yield, as shown for the case of a $4 \mathrm{~J}$ solar cell. They can also be applied to analyze the performance of the cell under any kind of situation where the spectral content of the light impinging on the cell deviates from a reference spectrum, such as when designing a receiver assembly where the optics have a significant dispersion in the transmission and that may change with time/tracking accuracy. A method to generate a reduced set of equivalent annual spectra for a site is being developed, which will speed up the energy yield measurement technique and enable its application to concentrator operation.

\section{REFERENCES}

[1] P. Faine, S. R. Kurtz, C. Riordan, and J. M. O1son, "The influence of spectral solar irradiance variations on the performance of selected single-junction and multijunction solar cells," Sol. Cells, vol. 31, no. 3, pp. 259-278, Jun. 1991.

[2] P. J. Verlinden and J. B. Lasich, "Energy rating of concentrator PV systems using multi-junction III-V solar cells," in Proc. 33 rd IEEE Photovoltaic Spec. Conf., 2008 , pp. 1-6.

[3] G. S. Kinsey and K. M. Edmondson, "Spectral response and energy output of concentrator multijunction solar cells," Prog. Photovoltaic Res. Appl., vol. 17 , no. 5, pp. 279-288, Aug. 2009.

[4] M. A. Steiner and J. F. Geisz, "Non-linear luminescent coupling in seriesconnected multijunction solar cells," Appl. Phys. Lett., vol. 100, no. 25, art. no. 251106 , Jun. 2012.

[5] A. S. Brown and M. A. Green, "Radiative coupling as a means to reduce spectral mismatch in monolithic tandem solar cell stacks theoretical 
considerations," in Proc. 29th IEEE Photovoltaic Spec. Conf., 2002, pp. 868-871.

[6] G. Peharz, G. Siefer, K. Araki, and A. W. Bett, "Spectrometric outdoor characterization of CPV modules using isotype monitor cells," in Proc. 33rd IEEE Photovoltaic Spec. Conf., 2008, pp. 1-5.

[7] M. Meusel, C. Baur, G. Siefer, F. Dimroth, A. W. Bett, and W. Warta, "Characterization of monolithic III-V multi-junction solar cellsChallenges and application," Sol. Energy Mater. Sol. Cells, vol. 90, nos. 18/19, pp. 3268-3275, Nov. 2006.

[8] K. A. Emery, C. R. Osterwald, T. W. Cannon, D. R. Myers, J. Burdick, T. Glatfelter, W. Czubatyj, and J. Yang, "Methods for measuring solar cell efficiency independent of reference cell or light source," in Proc. IEEE PV Spec. Conf., 1985, pp. 623-628.

[9] D. J. Friedman, J. F. Geisz, and M. A. Steiner, "Analysis of multijunction solar cell current-voltage characteristics in the presence of luminescent coupling," IEEE J. Photovoltaics, vol. 3, no. 4, pp. 1429-1436, Oct. 2013.

[10] J. F. Geisz, M. A. Steiner, I. García, S. R. Kurtz, and D. J. Friedman, "Enhanced external radiative efficiency for $20.8 \%$ efficient single-junction GaInP solar cells," Appl. Phys. Lett., vol. 103, no. 4, art. no. 041118, Jul. 2013.

[11] M. W. Wanlass, K. A. Emery, T. A. Gessert, G. S. Horner, C. R. Osterwald, and T. J. Coutts, "Practical considerations in tandem cell modeling," Sol. Cells, vol. 27, nos. 1-4, pp. 191-204, Oct. 1989.
[12] N. L. A. Chan, T. B. Young, H. E. Brindley, N. J. Ekins-Daukes, K. Araki, Y. Kemmoku, and M. Yamaguchi, "Validation of energy prediction method for a concentrator photovoltaic module in Toyohashi, Japan," Prog. Photovoltaic Res. Appl, vol. 21, no. 8, pp. 1598-1610, Dec. 2013.

[13] M. A. Steiner, J. F. Geisz, I. Garcia, D. J. Friedman, A. Duda, W. J. Olavarria, M. Young, D. Kuciauskas, and S. R. Kurtz, "Effects of internal luminescence and internal optics on and of III-V solar cells," IEEE J. Photovoltaics, vol. 3, no. 4, pp. 1437-1442, Oct. 2013.

[14] D. R. Myers, "Terrestrial solar spectral distributions derived from broadband hourly solar radiation data," Proc. SPIE, vol. 7410, pp. 74100A-174100A-11, 2009.

[15] R. M. France, J. F. Geisz, I. Garcia, M. A. Steiner, W. E. McMahon, D. J. Friedman, T. E. Moriarty, C. Osterwald, J. S. Ward, A. Duda, M. Young, and W. J. Olavarria, "Quadruple junction inverted metamorphic concentrator devices," IEEE J. Photovoltaics, DOI:10.1109/JPHOTOV.2014.2364132

[16] R. E. Bird and C. Riordan, "Simple solar spectral model for direct and diffuse irradiance on horizontal and tilted planes at the earth's surface for cloudless atmospheres," J. Clim. Appl. Meteorol., vol. 25, no. 1, pp. 87-97, Jan. 1986.

Authors' photographs and biographies not available at the time of publication. 